As regards the Entericus Subgroup it can be distinguished from $B$. giumai, Castellani, 1913, by not producing acid and gas in dextrin, and by producing acid and gas in glycerol, adonitol, dulcitol, and mannitol, and from $B$. enterious, Castellani, 1909, by not fermenting dextrin, and by producing acid and gas in adonitol and acidity in litmus milk.

We therefore conclude that it is a distinct species and we define it as follows. "A short, non-motile, homogeneous, non-encapsulated, Gram-negative bacillus with rounded ends, which produces a whitish, non-fluorescent growth under aerobic and anaerobic conditions upon agar-agar at $37^{\circ} \mathrm{C}$., and on gelatin, which is not liquefied, at $20^{\circ} \mathrm{O}$. and which is capable of producing acid and gas in glucose, lactose, adonitol, dulcitol, and mannitol, but not in saccharose, raffinose, or dextrin, and which gives rise to acidity without clot in litmus milk and is also an indol producer. It was isolated from the fæces of persons suffering from an entericlike fever in Khartoum and Omdurman."

It failed to agglutinate with a colon specific serum of high titre, but it showed group reactions with high-titre paratyphoid A and B specific sera. It is therefore related not merely to the Colon Subgroup but to the ParatyphoidGaertner Subgroup, and we consider it and possibly the whole Entericus Subgroup to be connecting links between these two collections of organisms.

As it was present in the patients' fæces during the illness and absent after recovery, and as it agglutinated with the patients' serum, even though we were unable to obtain it from the peripheral blood during the attack, we consider that it was the causal organism of the attacks of enterio-like fever, using this term in its broadest sense.

It gives us much pleasure to acknowledge the trouble taken by Dr. Papatheodorou, of Khartoum, and Dr. Muzhir, of Omdurman, in providing us with material for this paper.

References (Arranged in Alphabetical Order).-Alexander (1914) Supplement to the Annual Report of the Local Government Board, 384-400, London. Archibald (1911): Fourth Report Wellcome Tropical Research Laboratories, Medical, 319-334, London. Browne (1914) Journal of Infectious Diseases, xv., 580-604, Chicago. Castellan (1902-1914): Ceylon Medical Reports, Colombo; (1907) Journal of Hygiene, vii., 1-12, Cambridge; (1909-1913) Reports of the Advisory Commit tee for the Tropical Disease Fund, London; (1912) Journal of Trowical Medicine and Hygiene, xv., 164-165, Lnndon. Castellani and Chalmers (1910): Manual of Tropical Medicine, 991 ; (1913) Ibid., second edition, 1380-81, London. Clemensha, Aiyar, and Mudaliyar (1909). Bacteriology of Water Supplies in Tropical Climates, Madras. Fif zgerald (1914): Journal of Infectious Diseases, xv, 268-278, Chicago Jackson (1911) : Ibid., viii., 241-249. Kligler (1914) : Ibid., xiv., 81-86, 187-204. MacConkey (1905): Journal of Hroiene, v., 333-379; (1906) Ibid. vi , 385-407; (1909) lbid., ix,, 86-103. Cambridge. Perkins (1904): Journal of Infectious Diseases, i., 241-267, Chicago. Rogers, Clarke, Mansfield, Davis, and Brooke (1914): Ibid,, xiv., 411-475. Smith (1915): Mansfield, Davis, and Brooke (1914): Ibid.,
Brit. Med. Jour., ii., July 3rd, 1-5, London.

\section{THE GANGRENE OF WAR : GASEOUS CELLULITIS OR EMPHYSHMATOUS GANGRENE.}

BY ALFRED J. HULL, F.R.C.S. ENG., MAJOR, ROYAL ARMY MEDICAL CORPS.

THE most common form of gangrene met with in the present campaign is an acute infection due to the bacillus of malignant œedema, the Bacillus aërogenes or Bacillus perfringens. The actual cause of gangrene is the presence in the wound of the pathogenic organism which finds a suitable medium in which to grow in the lacerated and devitalised tissue of gunshot wounds. In its most typical and dangerous forms gas gangrene occurs associated with deep wounds, the infection being carried into the tissues by the missile which inflicts the wound. It is therefore a primary and deep infection. Once the wound is infected the occurrence of gangrene is favoured by several conditions: 1. Delay in cleansing the wound and removing the foreign body. 2. Excessive damage to the tissues and the presence of necrosed tissue and blood clot. 3. Interference with the blood-supply. The tension produced by the effused blood, the gas, and other products of inflammation produce a vicious circle. 4. The presence of pus-forming organisms. This virulent infection may attack any gunshot wound and forms one of the outstanding dangers to which injuries are subject in war. The complication of an injury by emphysematous gangrene is usually discovered two or three days after the injury, but it may begin within a few hours. A septic wound cannot be considered safe from its attack until the wound has become clean and healthy. Cases have occurred in comparatively small and unimportant wounds.

The following forms of the disease are fairly distinct, but it must be understood that these varieties are grades of the same disease, passing from one to another. They are grouped here because they form more or less distinct clinical entities requiring entirely different prognosis and treatment.

\section{Anaerobic Infeotion without Gaseous Cellulitis.}

In this type there is, as yet, no definite spreading cellulitis nor gas in the tissues, but there is discolouration of the skin and gas in the discharge from the wound with characteristic odour. This type, if untreated, goes on to gaseous cellulitis, of which it is really an early stage.

\section{Gaseons Cellulitis.}

This may be local or diffuse. The local variety is fairly amenable to treatment. The infection commences with a local cellulitis with a spread of gas in the tissues, and is usually associated with a deep septic wound. Compound fractures of large pipe-bones contribute a high proportion of the cases, and those with fracture of the femur yield the most fatal results. In the local form the cellulitis is limited to the neighbourhood of the wound and apparently has no tendency to spread to the rest of the limb. These cases react well to the simple treatment of multiple incision, and some would no doubt recover without special treatment. They account for the majority of successful cases and have made the reputation of several remedies. In the diffuse form the cellulitis involves the whole limb and often spreads to the deep tissues of the limb and the trunk. The skin around the wound is discoloured, but the more distant skin is unchanged. The skin may be mottled with blue, purplish, or grey blotches, but never presents the bright colour seen in ordinary cellulitis. The limb is very much swollen and is œdematous. The tissues which are permeated by the gas yield a crackling sensation to the touch similar to that found in surgical emphysema. The circulation in the hand or foot is not affected until quite late. The cellulitis appears to be mainly confined to the subcutaneous tissues, the gas penetrating the cellular tissue in advance of the cellulitis The patient's general condition is soon affected unless the cellulitis is limited to a small area. There is profound toxæmia with a rapid pulse of small volume, the hands and feet are cold, and vomiting and hiccough often occur. In spite of this the patient may not complain of feeling very ill

Treatment. - The treatment of the wound does not differ from the treatment of other septic gunshot wounds. The wound should be freely opened and all devitalised tissue excised. The missile should be removed and the wound searched for other septic matter, such as fragments of clothing. Very free drainage, in a dependent position, should be provided. The cellulitis, whether local or general, should be treated by numerous incisions into the subcutaneous tissues. In a local case, affecting the calf of the leg, three or four incisions, several inches in length, should be made down to, but not through, the deep fascia. In addition to these deep incisions half-a-dozen two-inch incisions through the skin, just entering the superficial fascia, should be made. The wound may be dressed with hypertonic saline solution. The infection being an anaerobic one and mainly limited to the cellular tissue, the injection of hydrogen peroxide into the cellular tissue at a point proximal to the cellulitis has been undertaken with a view to checking the advance of the disease. About 20 c.c. of a ten-volume solution is injected by means of a syringe into the circumference of the limb above the infection. The necessity for this is not established. Collapse, sloughing, and other bad results have followed this treatment. If the infection be localised and the patient's general condition good, arm or leg baths of peroxide of hydrogen may be given. Usually the limb is left exposed and continuous irrigation with 5 per cent. saline solution employed. If not effectively treated, the gaseous cellulitis may advance to a massive gangrene of the whole limb, the milder condition passing into the more fatal one with the greatest rapidity. The pulse becoming imperceptible in the extremity of the limb, the hand or foot becoming cold or numb, are indications for immediate amputation. 


\section{Looalised Gangrene.}

Around a septic wound a gaseous cellulitis occurs, and bubbles of gas may be detected in the pus coming from the wound. The skin surrounding the wound becomes mottledpurple in colour, and emphysematous crackling can be felt for a short distance beyond the gangrenous area. The case may remain one of localised gangrene or may be overcome by the infection and pass into a case of gangrene of the limb. Extremely foul, dirty fluid, containing bubbles of gas, exudes from the wound, but typical pus is absent or obscured by the gangrenous discharge. An orange-coloured discharge, which stains dressings, is sometimes present. A large number of wounds secrete pus or discharge containing bubbles of gas, although gas is not actually present in the tissues. 'These wounds recover if treated early by the ordinary method of treatment without extensive operation. In some cases the "gas " has been sucked in owing to movements of the limb. "The "gas" is not mixed with the pus in the same way in these cases and the characteristic odour is absent.

Treatment.-The devitalised tissues should be excised, and the usual eleansing and drainage operation should be performed on the wound if it has not already been done. Multiple incisions should be made in the emphysematous area. Hypertonic treatment gives good results. The discoloured area should be marked, and if after such treatment it is found to be spreading, amputation should be performed. If the hand or foot is found to be cold and numb and pulseless, it is dangerous to wait until further spread of the local gangrene takes place and amputation should be performed. Sometimes free incision of the calf will restore the circulation of the foot. In such cases the feeble circulation is due to the pressure of the exudate on the vessels under the deep fascia.

Illustrative case of localised gangrene (Fig. 1).--Sergeant-. wounded on March 2 nd with a fragment of high-explosive

FIG. 1.

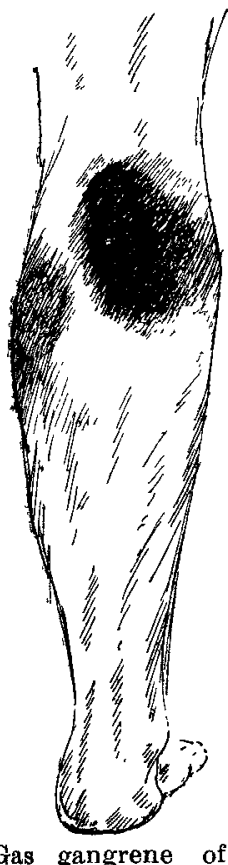

Gas gangrene of calf (localised) on outer side of leg outering leg, margins grey and sloughing, with area of con gestion around. shaded patch on back of leg representso areation, acute congestion, centre showing greyish slough, while lighter shading shows pateh on surface of skin. shell; entrance wound on the outer side of the leg about middle of fibula; no exit found. Wound dressed at field ambulance and patient arrived at base on March 4th. Leg swollen and painful and cedematous over tibia; large red patch over midale of calf, extending round to inner side of leg. $X$ rays showed shell fragment in calf muscles and comminuted fracture of fibula. Entrance wound was excised and shell fragment removed. Incisions were made over the inner side of the leg through extending red patch and in middle of calf. Small quantity of gas and pus escaped and calf muscles felt soft and spongy. The wounds were well irrigated with eusol and tubes were inserted. Temperature $100^{\circ} \mathrm{F}$. (fell to $98.4^{\circ}$ ); pulse 110 (fell to 96). The pus showed B. aëro genes capsulatus and streptococci. The patient made a good recovery.

\section{Massive Gangrene.}

This corresponds to the distribution of a vessel and may affect part or the whole of a limb. The organisms develop in the part which is rendered anæmic by damage to the blood-supply of the infected area. Examples of this type may be found in gangrene affecting the gluteal, rectus femoris, the adductor, and hamstring groups of muscles.

Illustrative case of massive gangrene (Fig. 2). Private — wounded on March 5th with shrapnel ; first and second metatarsals comminuted, shrapnel passing back, fracturing os calcis and having exit wound in heel. The wound was packed with cyanide gauze and the patient transferred to the base on March 7th. The foot was very much swollen and had two bullae on the dorsum. The inner side of the foot was black. with grey patches, while the ankle-joint was red and cedematous. There was a foul-smelling discharge containing gas from the wound, which contained $B$. aërogenes capsulatus. Amputation was performed at the middle third of the leg by the open method. The patient made an uninterrupted recovery.

\section{Gangrene of the Limb.}

The infection is usually apparent within the first three days after the receipt of the injury. Massive gangrene of the whole limb develops and the limb dies en masse. The process is extremely rapid, a marked change taking place within a few hours. On several occasions a limb which has been considered healthy in the evening has been discovered to be affected with diffuse gangrene the following morning.

The patient is in a highly toxic con

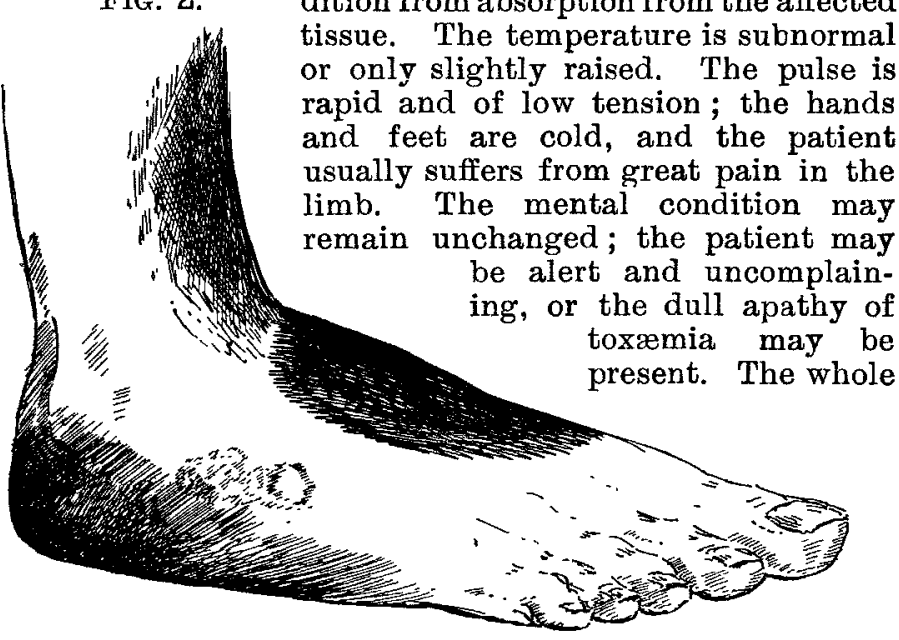

Gas gangrene of foot en masse. The dark shading on dorsum and inner side of foot shows the dead gangrenous area in the region of entrance wound, while around tbe exit wound in heel the same is represented. The bullæ are seen just below the ankle-joint, while the lighter shading extending up the leg marks an area of acute congestion.

limb becomes swollen and tense and no pulse can be felt in the extremity, and the hand or foot is cold and numb. The skin is at first grey-white, then mottled grey, green, yellow, purple, and finally black. The infection commences in the neighbourhood of a septic wound, often associated with a septic, compound, comminuted fracture. The gangrene spreads with great rapidity, the gas diffusing in the cellular tissues, especially beneath the skin and along the course of the great vessels. A crackling sensation can be obtained upon palpation above the actually gangrenous area of the limb, the subcutaneous, gaseous cellulitis rapidly spreading to the abdomen or chest. The patient may die of toxæmia before the limb has actually become gangrenous, or even before the gangrenous areas have appeared.

Illustrative case of gangrene of limb.-Private — was admitted to a base hospital suffering from a gunshot wound of the left arm. The wound was of the explosive type, probably inflicted at close range. The entrance wound was small; the exit wound, over 4 inches in diameter, was lacerated. There was a severely comminuted fracture of the humerus with great destruction of bone, and spicules of bone protruded from the ragged sloughing wound. A foul-smelling, dirty-grey coloured discharge containing bubbles of gas exuded from the wounds. Around the wound for several inches the tissues were black and gangrenous, the whole of the limb was in tensely swollen, massive gangrene extended up to the axilla, the skin on the inner side being of a mottled green. Above the gangrenous area, extending into the axilla and chest on either side and on to the shoulder above, crackling could be felt on pressure. The outer arm was mottled greyish-brown up to within 3 inches of the acromion. The forearm and hand were swollen with tense cedema, mottled greyish-green, cold, and pulseless. The patient's condition was very unfavourable; the pulse was small and running, the temperature was $100^{\circ} \mathbf{F}^{\prime}$, the tongue was dry and furred, and the face drawn and anxious. Open ether was administered, preceded by atropine gr. 1/100 and morphia gr. $\frac{1}{4}$. An intravenous saline injection was begun before the operation. The subcutaneous tissue above the gangrenous area was infiltrated with a 10-volume solution of peroxide of hydrogen. A circular incision was made on a level with the lower margin of the axilla on the inner side and about 3 inches below the acromion process on the outer side, the incision passing through tissue infected with gaseous cellulitis. The circular incision was carried right down to the bone, whilst the subclavian artery was compressed. After raising the deltoid, the humerus was disarticulated at the shoulder. The wound was left open and dressed with saline solution; no sutures were used. The patient suffered severely from shock after the operation. Rectal injections 
of saline were given during the night. After the first 48 hours' extreme prostration the patient's condition improved. Continuous saline irrigation was instituted, the wound being dressed only with a layer of gauze. A crescentic-shaped area of tissue which formed the outer flap became coal-black in colour. This piece of tissue demarcated immediately below the acromion and separated eight days later. The wound granulated and rapidly became healthy. It is possible that the sloughing of the flap was due to the inflitration with hydrogen peroxide. This has been omitted in more recent cases with equally good results. The shock would have been less in all probability had the amputation area been infiltrated with local anæsthetic solution.

\section{Metastatio Gaseous Gangrene.}

The above condition has been observed. The gas infection occurs at a part distant from the wound. This exceedingly rare form of infection is presumably a blood infection.

\section{Prevention and Treatment.}

The means of preventing gas gangrene will be gathered from its causation. An early cleansing and drainage operation and the removal of missiles, searching examination for concealed septic pieces of clothing, and the efficient drainage of wounds are the primary means of its prevention. The enlargement of apparently clean wounds in cases of fracture of large pipe-bones is advisable where there is any sign of tension and swelling. The interference with the circulation will be obviated by avoiding the use of tourniquets and tight bandages and by efficient splinting which corrects the deformity of fractures. The use of strong antiseptics is to be deprecated, and weak antiseptics will have no effect upon the resistant organisms.

The treatment of gas gangrene is dependent upon two principles. First, the drainage of subcutaneous tissue by means of free incisions, in order that the products of inflammation may escape and the spread of infection to the deeper parts may be prevented. The forms of gangrene limited to the cellular tissues will usually react well to this treatment. Secondly, the excision of all necrotic tissue. By excision of all necrotic tissue is meant that, irrespective of anatomical consideration, the necrotic area must be completely excised, and, in the more severe cases, amputation is the only possible mode of effecting complete excision.

When, in the presence of compound fracture, gas is found to be issuing from the wound and the skin is becoming grey and mottled and the hand or foot is cold and numb, amputation should be performed without delay, as it is of the utmost importance to amputate before the patient is dying of toxæmia. The disease is an extremely fatal one in any case, and calls for recognition and amputation at an early stage. The only form of amputation which can be considered is a direct circular amputation. The amputation should be performed above the infected tissue if possible. Some cases have recovered in which this has not been possible, the gaseous cellulitis having already spread to the trunk. Extension of gas to the trunk does not negative amputation. Amputation should, in such cases, be performed through the gas-infiltrated tissues, and the remaining infected tissues treated by free incisions. A tourniquet is applied, and in order to obviate shock as much as possible a circular infiltration of all the tissues is made around the whole circumference of the limb with novocaine adrenalin solution.

Several methods of combating the shock of an amputation are available, and, since the amputation for gaseous gangrene has to be performed when the patient is in a very toxic condition and very subject to shock, it is essential that one of them be employed. Infiltration of the cauda equina and sacral nerves by introducing 20 c.c. of novocaine solution slowly into the sacral canal through the terminal foramen, the horns of the last piece of the sacrum being the guide to the aperture, is a good method and strongly advocated by some surgeons. Blocking the main nerve trunks by injecting them with local anæsthetic solution is a well-known method. Spinal anæsthesia is perhaps the best method. I have found the local infiltration of the tissues to be divided, with special care in injecting the great nerve trunks, a most satisfactory method. No local ill effects or gangrene have followed the infiltration. Saline solution is usually given into a vein during the operation. All the tissues are divided at the level to which the skin retracts after the circular skin incision has been made. No sutures are inserted by this method and free drainage is obtained. The wound is dressed with hypertonic saline solution, applied on gauze. After the first few hours constant irrigation is applied to the gauze from an irrigator.

\section{CARDIAC SYMPTOMS FOLLOWING DYSENTERY AMONG SOLDIERS.}

BY E. B. GUNSON, M.D. EDIN., M.R.C.P. LOND., CAPTAIN, R.A.M.C., ATTACHED HAMPSTEAD MILTTARY HOSPITAL.

THE present paper is based upon notes of 65 consecutive cases of dysentery admitted to the 19th General Hospital, Alexandria, from Gallipoli in October, 1915. The cases were typical of several hundred admitted to the hospital over a period of several months.

Severity and nature of the disease.-Practically all the cases were examples of severe infection; all gave a history of the presence of blood in the stools persisting for several days, the blood still being present on admission in a large proportion of the patients. No bacteriological examination of the stools was carried out, but on clinical grounds 75 per cent. were judged to be amobic, the remainder bacillary in type.

Past history. - There was a history of rheumatic fever in 6 per cent.

Control of the patients.-After admission the worst cases, some of whom were unable to walk or stand owing to extreme exhaustion, were kept in bed entirely. The remainder, including some patients who were still passing blood, often had perforce to rise and walk to the lavatory, a distance of 20 yards from the ward. This necessitated rising, in some cases, as often as 30 times during the first 24 hours after admission, and the exertion called for in patients still profoundly weak must be regarded as an important factor in increasing the effect of the disease upon the heart. Even more important, probably, in this connexion is the fact that the majority of the patients continued in the trenches for many days after the onset of dysentery; they often reported sick only when they were completely exhausted.

Drugs. - All the patients received two courses of subcutaneous injections] of emetin, in half-grain doses, twice a day for four days. In the severe bacillary cases antidysenteric serum was used. Hæmorrhage from the bowel, diarrhoea, and pain were treated symptomatically.

\section{Circulatory System.}

The symptoms and physical signs varied with the severity of the disease.

A. Fatal oases.-There were three fatal cases in the present series, but many others were observed. The circulatory disturbance was profound. The patient was pale. The extremities were cold and clammy. The pulse was small and feeble, at times imperceptible at the wrist with a rate of about 100 per minute. Premature contractions (extrasystoles) were occasionally met with. Cardiac dilatation followed the extreme enfeeblement of the pulse within 24 or 48 hours in rapidly fatal cases and was accompanied by marked venous congestion, especially of the head and neck. In less rapid cases the cardiac dilatation and enfeeblement were delayed for several days after the peripheral circulation had first failed, as there were not infrequently marked remissions in the peripheral failure of which the state of the pulse and the cold, clammy extremities were evidence. There was usually slight enlargement of the liver. The condition of the patients presented a striking resemblance to that met with in toxic cases of diphtheria. The primary change was apparently a vaso-motor paralysis followed by cardiac dilatation and enfeeblement of heart sounds.

B. Non-tatal cases.-This group is of special interest, as in not a few cases of so-called "soldier's heart" the onset of symptoms dates definitely from an attack of dysentery.

1. Aoute stage. - In some cases a condition approximating to that described above was met with, and persisted for several days. In other cases the pulse remained of good strength and no signs of circulatory failure occurred.

2. Convalescent stage.-In practically all cases the symptoms of general weakness and exhaustion met with during convalescence from any severe infectious disease, when patients are first allowed up, were present. However, unlike many other diseases, convalescence from dysentery is associated in a number of cases with persistent symptoms of circulatory inefficiency, such as a sense of exhaustion, dyspnoea, palpitation, and giddiness after very moderate exertion, which lasts for several weeks and in some cases for several months. 